\title{
Ability of swept source OCT to detect retinal changes in patients with bipolar disorder
}

\author{
Vicente Polo ${ }^{1,2} \cdot$ Maria Satue $^{1,2} \cdot$ Alicia Gavin $^{1,2} \cdot$ Elisa Vilades $^{1,2} \cdot$ Elvira Orduna $^{1,2} \cdot$ Marta Cipres $^{1,2}$. \\ Javier Garcia-Campayo ${ }^{2,3} \cdot$ Mayte Navarro-Gil $^{4}$ Jose M. Larrosa ${ }^{1,2} \cdot$ Luis E. Pablo $^{1,2}$ - Elena Garcia-Martin ${ }^{1,2}$
}

Received: 27 November 2017 / Revised: 15 May 2018 / Accepted: 4 June 2018 / Published online: 31 October 2018

(c) The Royal College of Ophthalmologists 2018

\begin{abstract}
Purpose To evaluate the ability of swept source optical coherence tomography (SS-OCT) to detect retinal changes in patients with bipolar disorder (BD).

Methods Twenty-three patients with BD and 23 controls underwent retinal evaluation using SS deep range imaging (DRI) Triton OCT. Full retinal thickness, the ganglion cell layer (GCL), the retinal nerve fiber layer (RNFL), and choroidal thickness were evaluated with automated segmentation software.

Results Patients with BD were shown to have significant thinning of the macular full retinal thickness in the center $(p=$ $0.049)$, inner temporal ( $p=0.045)$, inner nasal $(p=0.016)$, and inner inferior $(p=0.016)$ of the ETDRS areas. The macular GCL layer was reduced in patients compared with controls (average, $p=0.002$; superior, $p=0.009$; superonasal, $p=0.009$; inferonasal, $p=0.003$; and inferior, $p=0.009$ ). Peripapillary reduction of full retinal thickness (average, $p<0.001$; superotemporal, $p<0.001$; superonasal, $p=0.003$; nasal, $p=0.005$; and inferotemporal, $p=0.033$ ), GCL (nasal, $p=$ 0.025 ), and RNFL thickness (average, $p=0.002$; superotemporal, $p<0.001$; and superonasal, $p=0.045$ ) was observed in patients compared with controls. No significant differences were observed in choroidal thickness measurements.

Conclusions BD patients were shown to have quantifiable thinning of full retinal thickness and the GCL in the macular area, as well as a peripapillary reduction of the RNFL and GCL thickness. The analysis of the retinal sublayers with SS-OCT may be a useful indicator to show degeneration and monitor disease progression in bipolar disorder.
\end{abstract}

\section{Introduction}

In the last decade, the structural analysis of the retina using optical coherence tomography (OCT) has demonstrated to

Electronic supplementary material The online version of this article (https://doi.org/10.1038/s41433-018-0261-6) contains supplementary material, which is available to authorized users.

Maria Satue

mariasatue@gmail.com

1 Department of Ophthalmology, Miguel Servet University Hospital, Zaragoza, Spain

2 Aragon Health Research Institute (IIS Aragon, IACS), Zaragoza, Spain

3 Department of Psychiatry, Miguel Servet University Hospital, Zaragoza, Spain

4 Department of Psychology and Sociology, Faculty of Social and Human Sciences, University of Zaragoza, Zaragoza, Spain be an excellent tool for detecting and monitoring axonal loss and neurodegeneration in patients with neurodegenerative diseases $[1,2]$. Very recently, psychiatric disorders, such as fibromyalgia and schizophrenia have shown to cause objective retinal changes and axonal loss as observed with OCT [3, 4]. Based on these recent findings, OCT might also become an essential tool in the diagnosis and follow-up of patients with psychiatric diseases.

Bipolar disorder (BD) is a chronic psychiatric disorder that alternates episodes of mania with episodes of depression. The prevalence of $\mathrm{BD}$ is estimated at $1 \%$ of the general population. However, some recent studies have demonstrated a higher prevalence (>5\%) [5], possibly explained by the long delay in diagnosis of this disorder [6]. Literature on the structural retinal analysis in BD patients is very recent and scarce. We could only find three published studies demonstrating axonal loss in the retina of these patients using spectral domain OCT, with somewhat contradictory results [7-9]. We could not find any published 
study on the applicability of swept source (SS) technology in the evaluation of retinal changes in psychiatric disorders.

The objective of the present study is to evaluate retinal changes in patients with BD using new SS-OCT technology, providing an analysis of the different retinal layers.

\section{Methods}

Patients with confirmed BD were included in an observational cross-sectional study. The sample size was calculated in order to detect significant differences in the retinal nerve fiber layer (RNFL), assuming an $\alpha$ error of $5 \%$ and a $\beta$ error of $10 \%$. Based on these calculations, the number of subjects needed was at least 20 . A total of 23 patients with BD and 23 age- and sex-matched healthy individuals were recruited for the study. All procedures adhered to the tenets of the Declaration of Helsinki, and the local ethics committee (CEICA, ethics committee for scientific research in Aragon) approved the experimental protocol. All participants provided informed consent to participate in the study.

The diagnosis of BD was established by a trained psychiatrist based on the Diagnostic and Statistical Manual of Mental Disorders, 4th edition (DSM-IV) criteria [10]. The Young Mania Rating Scale (YMRS) and the Hamilton Depression Rating Scale (Ham-D) (Hamilton, 1960) were applied to confirm euthymic status in all patients. Smoking habit, prescribed treatments, and disease duration were recorded.

Inclusion criteria were: confirmed BD diagnosis; bestcorrected visual acuity (BCVA) of +0.4 Log MAR or lower in each eye to allow for performance of the protocol; and intraocular pressure $<21 \mathrm{mmHg}$. Exclusion criteria were: the presence of significant refractive errors ( $>5$ diopters of spherical equivalent refraction or 3 diopters of astigmatism); intraocular pressure $\geq 21 \mathrm{mmHg}$; media opacifications; concomitant ocular diseases, including history of glaucoma or retinal pathology; and systemic conditions that could affect the visual system. Healthy controls had no history nor evidence of ocular or neurologic disease of any nature; their BCVA was $\leq 0.2 \log$ MAR. Only one eye per subject was randomly selected and included.

All subjects underwent a complete neuro-ophthalmic evaluation that included pupillary, anterior segment, and funduscopic examination. Structural analysis of the retina was performed using SS-OCT technology with the deep range imaging (DRI) Triton OCT (Topcon, Japan). The Triton SS-OCT uses a tunable laser as a light source to provide a $1050 \mathrm{~nm}$ centered wavelength, reaching a scanning speed of 100,000 A-scans per s and yielding 8 and $20 \mu \mathrm{m}$ axial and transverse resolution in tissue, respectively.

In this study, we utilized the $3 \mathrm{D}(\mathrm{H})$ Macula +5 Line Cross protocol. This protocol performed a $12.0 \times 9.0 \mathrm{~mm}$
3D scan plus a double $9.0 \mathrm{~mm}$ radial scan (with an overlap of eight linear scans in the center) of the macular and peripapillary area providing a fast and complete evaluation of a wide retinal area in a single scan capture. The results of the area analyzed by this protocol are displayed as measurements of the nine macular areas of the early treatment diabetic retinopathy study (ETDRS scan, which includes a central $1 \mathrm{~mm}$ circle representing the fovea, inner and outer rings measuring 3 and $6 \mathrm{~mm}$ in diameter, respectively, central and average thickness plus macular volume), six macular sectors, and 4-12 sectors of the peripapillary area (TSINT scan: temporal-superior-inferior-nasal-temporal scan). The ETDRS scan includes measurements of the full retina and thickness of the choroidal plexus in the macular area. The six macular sectors provide additional data of the ganglion cell layer (GCL+, between the RNFL and the inner nuclear layer boundaries; $\mathrm{GCL}++$, between the inner limiting membrane -ILM- and the inner nuclear layer boundaries) of six macular areas (superotemporal, temporal, inferotemporal, superonasal, nasal, and inferonasal). The peripapillary area evaluated by the TSNIT scan provides information of the different morphometric data of the optic disc head (disc, cup, and rim areas; cup and ring volume; cup-disc ratios and disc diameters) and measurements of the full retina, RNFL, GCL,$+ \mathrm{GCL}++$, and choroidal thickness of 4 quadrants (superior, nasal, inferior, and temporal), 6 sectors (superonasal, superotemporal, nasal, temporal, inferonasal, and inferotemporal), and 12-clock sectors. The 12-clock sector measurements were not analyzed in this study to avoid redundant data. The data corresponding to the 4-quadrant analysis will not be shown in the tables.

The DRI Triton SS-OCT provides a quality scale in the image to indicate the signal strength. The quality score ranges from 0 (poor quality) to 100 (excellent quality). All scans were obtained by the same experienced operator and only images with a score $>55$ were analyzed in our study. Poor quality images prior to data analyses were rejected.

All data analyses were performed using SPSS software version 20.0 (SPSS Inc., Chicago, IL). The KolmogorovSmirnov test was used to assess sample distribution. Due to the normal distribution of the data, differences between evaluations of BD patients and healthy subjects were compared using the Student's $t$-test. The correlations between disease duration and OCT measurements (retinal thicknesses in nine ETDRS areas, average thickness, and total volume; GCL + layer thicknesses in six macular sectors; and peripapillary RNFL and GCL+ layer thicknesses in six TSNIT sectors) were also evaluated, using Pearson's correlation test. Age, sex, intraocular pressure (IOP), the smoking habit, and the morphometric parameters of the optic disc head were considered as confounding factors. All data were treated based on intention-to-treat analysis. $p$ values $<0.05$ were considered to indicate statistical 
significance. To avoid a high false-positive rate, the Bonferroni correction for multiple tests was calculated and the corrected $p$ values were added to the previously calculated data. Results of calculations are reported as "mean" and "SD" (see all Tables).

\section{Results}

Twenty-three eyes of 23 patients with a mean age of 49.67 years $(\mathrm{SD}=8.75)$, and 23 eyes of 23 healthy individuals with a mean age of 49.03 years $(\mathrm{SD}=9.44)$ were included in the study. There were no differences in age $(p=0.221)$ and intraocular pressure $(p=0.672)$. The male/female ratio was equal for both groups ( 8 males, 15 females). Mean spherical equivalent was $0.19 \pm 1.76 \mathrm{D}$ in the patients group and $0.18 \pm 1.80 \mathrm{D}$ in the control group $(p=0.578)$. The smokers rate was calculated for both groups; $60 \%$ of patients with BD were smokers (or had been for the past 5 years), $56.52 \%$ of the individuals in the control group were current (or had been for the past 5 years) smokers; ( $p=$ 0.065). No significant differences were observed in morphometric data of the optic disc head between patients with $\mathrm{BD}$ and controls $(p>0.05$ in all parameters provided by the protocol used: disc, cup, and rim areas, cup and rim volumes, cup/disc area ratio, linear and vertical cup/disc ratios, vertical and horizontal disc diameters). In patients group, disease duration was 16.12 years $(\mathrm{SD}=6.66)$, and the treatment analysis revealed that $78.2 \%$ were treated with lithium, $17.4 \%$ with valproate, and $4.3 \%$ were not under any current treatment.

\section{ETDRS macular analysis}

Patients with BD presented significant retinal thinning in the center $(234.87 \pm 15.72 \mu \mathrm{m}$ in patients vs $255.83 \pm 47.13 \mu \mathrm{m}$ in controls, $p=0.049)$, inner temporal $(293.06 \pm 11.05 \mu \mathrm{m}$ vs $306.20 \pm 28.47 \mu \mathrm{m}$, respectively, $p=0.045$ ), inner nasal (306.12 $\pm 10.06 \mu \mathrm{m}$ vs $320.46 \pm 25.67 \mu \mathrm{m}, p=0.016)$, and inner inferior $(301.48 \pm 15.62 \mu \mathrm{m}$ vs $314.02 \pm 18.19 \mu \mathrm{m}, p$ $=0.016$ ) of the ETDRS areas (Table 1, Fig. 1).

The analysis of the macular choroidal thickness showed no significant differences between patients with BD and healthy subjects.

\section{Six macular sector analysis}

Significant thinning of the macular GCL+ layer was observed in patients compared with controls, in the average $(70.13 \pm 3.40 \mu \mathrm{m}$ in patients vs $74.30 \pm 4.83 \mu \mathrm{m}$ in controls, $p=0.002)$, superior $(69.66 \pm 4.71 \mu \mathrm{m}$ vs $74.60 \pm 7.19 \mu \mathrm{m}$, respectively, $p=0.009)$, superonasal $(72.71 \pm 5.16 \mu \mathrm{m}$ vs $77.13 \pm 5.80 \mu \mathrm{m}, p=0.009)$, inferonasal $(71.62 \pm 3.97 \mu \mathrm{m}$
Table 1 Macular full retinal thickness and choroidal thickness measured with swept source deep range imaging Triton optical coherence tomography in bipolar disorder patients and controls

\begin{tabular}{|c|c|c|c|}
\hline $\begin{array}{l}\text { Macular ETDRS } \\
\text { measurements }\end{array}$ & Patients & Controls & $p$ \\
\hline \multicolumn{4}{|c|}{ Full retinal thickness $(\mu \mathrm{m}(\mathrm{SD}))$} \\
\hline Center & $234.87(15.72)$ & $255.83(47.13)$ & 0.049 \\
\hline Inner temporal & $293.06(11.05)$ & $306.20(28.47)$ & 0.045 \\
\hline Inner superior & 306.38 (10.94) & $\begin{array}{l}324.54 \\
(49.094)\end{array}$ & 0.090 \\
\hline Inner nasal & $306.12(10.06)$ & $320.46(25.67)$ & 0.016 \\
\hline Inner inferior & $301.48(15.62)$ & 314.02 (18.19) & 0.016 \\
\hline Outer temporal & $254.36(11.59)$ & $265.51(31.31)$ & 0.117 \\
\hline Outer superior & $270.24(8.52)$ & $290.05(70.27)$ & 0.187 \\
\hline Outer nasal & $28.07(8.92)$ & 298.97 (33.29) & 0.105 \\
\hline Outer inferior & $257.51(21.15)$ & $267.30(22.28)$ & 0.134 \\
\hline Avg thickness & $274.10(8.49)$ & $287.51(35.23)$ & 0.083 \\
\hline Center thickness & $194.04(22.45)$ & $213.60(73.47)$ & 0.228 \\
\hline $\begin{array}{l}\text { Total volume }\left(\mathrm{mm}^{3}\right. \\
(\mathrm{SD}))\end{array}$ & $1.02(0.24)$ & $8.13(1.02)$ & 0.086 \\
\hline \multicolumn{4}{|c|}{ Choroidal thickness $(\mu \mathrm{m}(\mathrm{SD}))$} \\
\hline Center & $\begin{array}{l}262.67 \\
(105.92)\end{array}$ & $\begin{array}{l}286.32 \\
(100.86)\end{array}$ & 0.442 \\
\hline Inner temporal & $257.68(90.22)$ & $281.66(95.37)$ & 0.386 \\
\hline Inner superior & $265.13(93.85)$ & 278.93 (95.08) & 0.622 \\
\hline Inner nasal & $\begin{array}{l}248.85 \\
(107.67)\end{array}$ & $\begin{array}{l}257.69 \\
(103.66)\end{array}$ & 0.778 \\
\hline Inner inferior & $250.93(95.65)$ & $\begin{array}{l}281.19 \\
(116.27)\end{array}$ & 0.340 \\
\hline Outer temporal & $241.99(74.15)$ & $264.14(83.83)$ & 0.348 \\
\hline Outer superior & $268.43(90.65)$ & $281.30(93.44)$ & 0.638 \\
\hline Outer nasal & $\begin{array}{l}212.58 \\
(103.05)\end{array}$ & 202.25 (97.04) & 0.728 \\
\hline Outer inferior & $249.79(98.32)$ & $260.10(93.45)$ & 0.717 \\
\hline Avg thickness & $246.51(87.65)$ & $258.16(86.26)$ & 0.652 \\
\hline Center thickness & $\begin{array}{l}264.17 \\
(110.09)\end{array}$ & $\begin{array}{l}287.47 \\
(101.72)\end{array}$ & 0.460 \\
\hline $\begin{array}{l}\text { Total volume }\left(\mathrm{mm}^{3}\right. \\
(\mathrm{SD}))\end{array}$ & $6.97(2.47)$ & $7.29(2.43)$ & 0.657 \\
\hline
\end{tabular}

Bold letters indicate $p$ value $<0.05$. Bonferroni corrections for multiple comparisons was calculated at $(p<0.004)$

ETDRs early treatment diabetic retinopathy study

vs $76.31 \pm 5.79 \mu \mathrm{m}, p=0.003)$ and inferior $(65.31 \pm 8.50$ $\mu \mathrm{m}$ vs $71.22 \pm 6.07 \mu \mathrm{m}, p=0.009$ ) sectors (Table 2). Significant changes were also observed in macular GCL ++ as described in Table 2 and Fig. 1.

\section{TSINT peripapillary analysis}

Patients presented significant thinning of peripapillary full retinal thickness in the average thickness $(289.70 \pm 11.46$ 


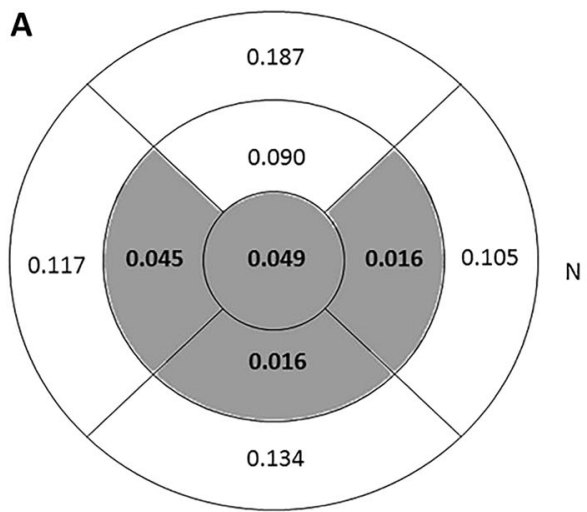

Fig. 1 Schematic representation of the macular changes measured by Triton OCT in patients with bipolar disorder. Gray color represents affected areas. $N$ indicates the nasal sector. The numbers show the level of significance; bold letters indicate $p<0.05$. a Full retinal

Table 2 Macular ganglion cell measurements measured with swept source deep range imaging Triton optical coherence tomography in patients with bipolar disorder and healthy controls

\begin{tabular}{llll}
\hline & Patients & Controls & $p$ \\
\hline Macular GCL+ $(\mu \mathrm{m}(\mathrm{SD}))$ & & \\
Average & $70.13(3.40)$ & $74.30(4.83)$ & $\mathbf{0 . 0 0 2} *$ \\
Superotemporal & $70.48(3.51)$ & $73.23(7.23)$ & 0.108 \\
Superior & $69.66(4.71)$ & $74.60(7.19)$ & $\mathbf{0 . 0 0 9}$ \\
Superonasal & $72.71(5.16)$ & $77.13(5.80)$ & $\mathbf{0 . 0 0 9}$ \\
Inferonasal* & $71.62(3.97)$ & $76.31(5.79)$ & $\mathbf{0 . 0 0 3} *$ \\
Inferior & $65.31(8.50)$ & $71.22(6.07)$ & $\mathbf{0 . 0 0 9}$ \\
Inferotemporal & $70.98(4.09)$ & $73.53(7.47)$ & 0.158 \\
Macular GCL++ $(\mu \mathrm{m})$ & $(\mathrm{SD}))$ & & \\
Average & $105.79(5.26)$ & $115.76(25.31)$ & 0.071 \\
Superotemporal & $94.20(3.84)$ & $106.23(41.34)$ & 0.172 \\
Superior & $107.05(5.72)$ & $123.21(55.83)$ & 0.174 \\
Superonasal & $117.65(7.11)$ & $134.16(52.67)$ & 0.143 \\
Inferonasal & $117.31(7.93)$ & $124.06(7.68)$ & $\mathbf{0 . 0 0 5}$ \\
Inferior & $102.40(14.92)$ & $108.36(6.62)$ & 0.087 \\
Inferotemporal & $95.93(5.49)$ & $99.91(7.28)$ & $\mathbf{0 . 0 4 2}$ \\
\hline B &
\end{tabular}

Bold letters indicate $p$ value $<0.05$. Asterisks mark significant values according to Bonferroni corrections for multiple comparisons $(p<$ $0.007)$

$G C L$ ganglion cell layer

$\mu \mathrm{m}$ in patients vs $303.26 \pm 10.82 \mu \mathrm{m}$ in controls, $p<0.001)$ and in the superotemporal $(315.72 \pm 14.56 \mu \mathrm{m}$ vs $342.51 \pm$ $16.76 \mu \mathrm{m}$, respectively, $p<0.001)$, superonasal $(299.11 \pm$ $22.30 \mu \mathrm{m}$ vs $320.45 \pm 23.84 \mu \mathrm{m}, p=0.003)$, nasal $(268.50$ $\pm 13.86 \mu \mathrm{m}$ vs $279.43 \pm 10.98 \mu \mathrm{m}, p=0.005)$, and inferotemporal $(318.58 \pm 24.47 \mu \mathrm{m}$ vs $333.32 \pm 20.90 \mu \mathrm{m}, p=$ 0.033 ) sectors (Table 3 ). The 4-quadrant analysis of the full retina revealed significant thinning of the superior (305.51 $\pm 15.67 \mu \mathrm{m}$ in patients vs $329.33 \pm 15.55 \mu \mathrm{m}$ in controls, $p$

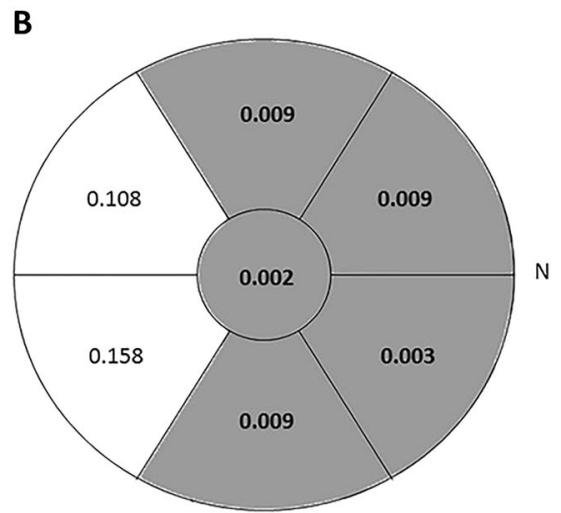

thickness displayed in nine ETDRS areas. b Ganglion cell layer thickness $(\mathrm{GCL}+)$ displayed in six macular sectors and average thickness (central area). OCT optical coherence tomography, ETDRS early treatment diabetic retinopathy study. (color figure online)

$<0.001)$, inferior $(305.69 \pm 21.06 \mu \mathrm{m}$ vs $320.64 \pm 14.22 \mu \mathrm{m}$, respectively, $p=0.011)$, and nasal quadrants (265.02 \pm $14.22 \mu \mathrm{m}$ vs $274.63 \pm 10.89 \mu \mathrm{m}, p=0.014)$ in patients compared to controls.

Significant thinning of the GCL+ thickness was observed in patients group in the nasal sector $(42.50 \pm$ $4.67 \mu \mathrm{m}$ in patients vs $45.41 \pm 3.77 \mu \mathrm{m}$ in controls, $p=$ 0.025). The GCL++ of BD patients presented significant thinning in the average thickness $(147.90 \pm 7.52 \mu \mathrm{m}$ vs $158.39 \pm 9.68 \mu \mathrm{m}, p<0.001)$, superotemporal $(174.68 \pm$ $16.29 \mu \mathrm{m}$ vs $198.89 \pm 18.12 \mu \mathrm{m}, p<0.001)$, superonasal $(158.65 \pm 21.18 \mu \mathrm{m}$ vs $174.60 \pm 23.16 \mu \mathrm{m}, \quad p=0.019)$, nasal $(128.60 \pm 10.97 \mu \mathrm{m}$ vs $135.85 \pm 12.16 \mu \mathrm{m}, \quad p=$ $0.039)$, and inferotemporal ( $183.61 \pm 22.00 \mu \mathrm{m}$ vs 196.65 $\pm 19.83 \mu \mathrm{m}, p=0.041$ ) sectors. (Table 3, Fig. 2) The 4quadrant analysis of the GCL ++ revealed significant thinning of the superior $(129.91 \pm 10.22 \mu \mathrm{m}$ vs $185.02 \pm$ $14.43 \mu \mathrm{m}, p<0.001)$ and inferior quadrants $(172.52 \pm$ $19.47 \mu \mathrm{m}$ vs $184.10 \pm 17.72 \mu \mathrm{m}, p=0.041$ ) in patients compared to controls.

The RNFL thickness of patients with BD presented significant thinning in the average thickness $(101.85 \pm 8.42$ $\mu \mathrm{m}$ in patients vs $110.42 \pm 9.18 \mu \mathrm{m}$ in controls, $p=0.002$ ) and the superotemporal $(131.80 \pm 17.80 \mu \mathrm{m}$ vs $155.49 \pm$ $19.18 \mu \mathrm{m}$, respectively, $p<0.001)$ and superonasal (114.24 $\pm 18.43 \mu \mathrm{m} \quad$ vs $127.78 \pm 25.47 \mu \mathrm{m}, \quad p=0.045) \quad$ sectors (Table 3, Fig. 2). The 4-quadrant analysis of the RNFL revealed significant thinning of the superior quadrant $(122.04 \pm 10.86 \mu \mathrm{m}$ vs $140.03 \pm 14.44 \mu \mathrm{m}, p<0.001)$ in patients compared to controls.

No significant differences were observed between patients and controls in the peripapillary choroidal thickness.

The correlation analysis revealed a significant inverse association between disease duration and full retinal 
Table 3 Peripapillary measurements obtained with swept source deep range imaging Triton optical coherence tomography in patients with bipolar disorder and healthy controls

\begin{tabular}{|c|c|c|c|}
\hline & Patients & Controls & $p$ \\
\hline \multicolumn{4}{|c|}{ Full retinal thickness $(\mu \mathrm{m}(\mathrm{SD}))$} \\
\hline Average & $289.70(11.46)$ & $303.26(10.82)$ & $<0.001 *$ \\
\hline \multicolumn{4}{|l|}{ Sectors $(\times 6)$} \\
\hline Superotemporal & $315.72(14.56)$ & $342.51(16.76)$ & $<0.001 *$ \\
\hline Superonasal & $299.11(22.30)$ & $320.45(23.84)$ & $0.003 *$ \\
\hline Nasal & $268.50(13.86)$ & $279.43(10.98)$ & $0.005 *$ \\
\hline Inferotemporal & $318.58(24.47)$ & $333.32(20.90)$ & $\mathbf{0 . 0 3 3}$ \\
\hline Inferonasal & $299.60(28.79)$ & $315.48(27.11)$ & 0.061 \\
\hline Temporal & $282.58(10.81)$ & $288.31(10.36)$ & 0.073 \\
\hline \multicolumn{4}{|c|}{ Peripapillary GCL $+(\mu \mathrm{m}(\mathrm{SD}))$} \\
\hline Average & $46.04(5.57)$ & $47.96(2.12)$ & 0.131 \\
\hline \multicolumn{4}{|l|}{ Sectors $(\times 6)$} \\
\hline Superotemporal & $42.87(7.85)$ & $43.40(7.57)$ & 0.819 \\
\hline Superonasal & $44.41(6.99)$ & $46.81(6.23)$ & 0.226 \\
\hline Nasal & $42.50(4.67)$ & $45.41(3.77)$ & 0.025 \\
\hline Inferotemporal & $45.36(11.23)$ & $44.73(4.25)$ & 0.803 \\
\hline Inferonasal & $40.00(12.61)$ & $44.54(5.40)$ & 0.121 \\
\hline Temporal & $55.46(5.91)$ & $56.58(4.99)$ & 0.494 \\
\hline \multicolumn{4}{|c|}{ Peripapillary GCL $++(\mu \mathrm{m})$} \\
\hline Average & $147.90(7.52)$ & $158.39(9.68)$ & $<0.001 *$ \\
\hline \multicolumn{4}{|l|}{ Sectors $(\times 6)$} \\
\hline Superotemporal & $174.68(16.29)$ & $198.89(18.12)$ & $<0.001 *$ \\
\hline Superonasal & $158.65(21.18)$ & $174.60(23.16)$ & 0.019 \\
\hline Nasal & $128.60(10.97)$ & $135.85(12.16)$ & $\mathbf{0 . 0 3 9}$ \\
\hline Inferotemporal & $183.61(22.00)$ & $196.65(19.83)$ & 0.041 \\
\hline Inferonasal & $168.10(31.60)$ & $178.92(29.55)$ & 0.237 \\
\hline Temporal & $129.91(10.22)$ & $134.31(8.44)$ & 0.118 \\
\hline \multicolumn{4}{|l|}{ RNFL $(\mu \mathrm{m})$} \\
\hline Average & $101.85(8.42)$ & $110.42(9.18)$ & $0.002 *$ \\
\hline \multicolumn{4}{|l|}{ Sectors $(\times 6)$} \\
\hline Superotemporal & $131.80(17.80)$ & $155.49(19.18)$ & $<0.001 *$ \\
\hline Superonasal & $114.24(18.43)$ & $127.78(25.47)$ & 0.045 \\
\hline Nasal & $86.09(11.33)$ & $90.43(12.54)$ & 0.225 \\
\hline Inferotemporal & $138.24(27.95)$ & $151.91(22.03)$ & 0.072 \\
\hline Inferonasal & $128.09(38.30)$ & $134.30(33.27)$ & 0.555 \\
\hline Temporal & 74.43 (11.44) & $77.73(10.82)$ & 0.322 \\
\hline
\end{tabular}

Bold letters indicate $p$ value $<0.05$. Asterisks mark significant values according to Bonferroni corrections for multiple comparisons $(p<0.007)$

$G C L$ ganglion cell layer, $R N F L$ retinal nerve fiber layer

thickness in the outer temporal $(r:-0.50, p=0.015)$ and inner inferior $(r:-0.43, p=0.043)$ sectors of the macular area (ETDRS areas), and also the inferotemporal sector of the peripapillary RNFL thickness $(r=-0.43, p=0.039)$ in
BD patients (see Supplementary tables 1 and 2 for complete results).

\section{Discussion}

To the best of our knowledge, this is the first study evaluating retinal changes in $\mathrm{BD}$ patients using the recent SS technology for OCT. In the present study, we assessed retinal changes in different macular and peripapillary layers in 23 patients with BD and 23 healthy controls. Significant thinning of the macular layers was observed in our patients, affecting the superior and inferior quadrants. The nasal sector was found to be more affected than the temporal sector in these patients.

Published studies on retinal changes in BD patients are scarce, especially those analyzing macular alterations. We could only find two published studies in which macular layers were assessed using spectral domain technology, and results were far from complete. Kalenderoglu et al. [7] found a significant reduction in the macular volume corresponding to the GCL in patients with BD. However, neither GCL thickness nor full retinal thickness were evaluated in their study. In a recent study by Khalil et al. [8], BD patients had a reduction of the GCL thickness in the superior quadrant and average thickness. Their results are consistent with our own results, considering that our patients presented significant reduction of the macular GCL thickness in the average and superior sector but also in the superonasal, nasal, and inferonasal sectors. This focal GCL changes may reflect focal affectation of the visual pathway in BD patients, as was observed previously in other neurological processes [11]. However, variability in GCL sector alteration is observed when our results are compared to similar published studies [7,8], and findings in our study might probably be reflecting a global loss of ganglion cells masked by the low statistical power due to a small sample size.

We could not find any changes in the total macular volume; however, GCL volume analysis was not performed by the Triton OCT, so it was not possible to corroborate Kalenderoglu's findings. Triton OCT provides measurements of macular full retinal thickness displayed as the ETDRS rings. However, GCL analysis is displayed as six macular sectors. Thus, both analyses are needed for a complete interpretation of the results provided by Triton OCT. The differences between both analyses (full retina and GCL) also indicate the importance of a complete retinal layer evaluation when assessing patients with $\mathrm{BD}$ and other neurodegenerative processes [12], since total macular thickness might not truly reflect the pathological changes of the inner layers. In our study, results from macular GCL+ measurements showed significant changes in the nasal, 


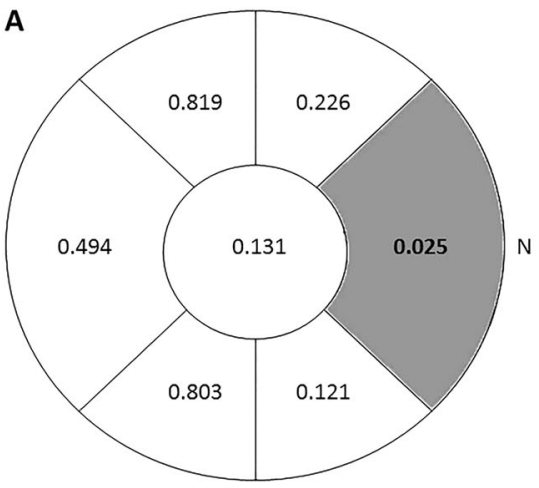

B
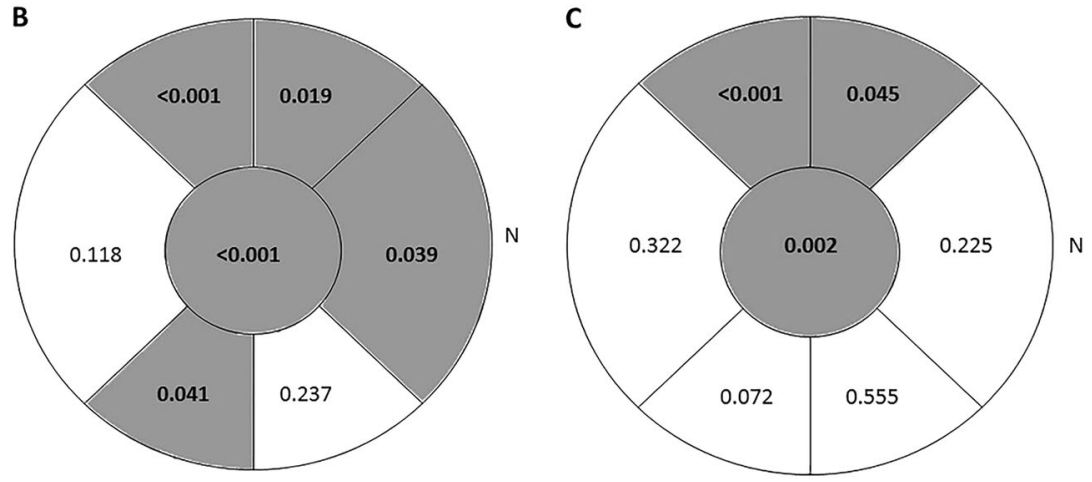

Fig. 2 Schematic representation of the peripapillary changes measured by Triton OCT in patients with bipolar disorder. Measured areas are displayed in six sectors (superonasal, nasal, inferonasal, inferotemporal, temporal, and superotemporal) and average thickness (central area). Gray color represents affected areas. $N$ indicates the

superior, and inferior sectors. Full retinal thinning however, only showed to be significant in the inner nasal and inner inferior ETDRS areas (corresponding to inferonasal measurements of the GCL+ thickness). Despite the scanned sectors do not exactly correspond between both protocols; we believe more retinal layers are involved (thinned) in this disease and may be responsible for the full retinal thickness changes observed. Further studies analyzing more separate retinal layers should be performed to corroborate this finding.

Triton OCT provided a complete analysis of the different peripapillary retinal layers in our patients, analyzing full retinal thickness, the GCL, and RNFL thickness. The average thickness, superior, and inferior areas were most affected in our patients, and surprisingly, the nasal quadrant was more affected than the temporal quadrant (as observed in the macular measurements). The analysis corresponding to the GCL++ (which includes the RNFL and the GCL) showed significant thinning in more areas (superonasal, superotemporal, nasal, inferotemporal, and average, the same results as the full retinal thickness measurements) than the analyses of the RNFL and GCL alone. This fact again shows the importance of using the segmentation analysis of the retinal layers, since full thickness alone may not reflect the complete picture, and a deeper analysis (using both separate layers and measurements of combined layers) might add important information about changes in the different retinal layers. Once more, published studies on peripapillary measurements in BD patients are scarce. Previously mentioned studies by Kalenderoglu and Khalil analyzed both macular and peripapillary areas [7, 8]. We found a third recent study by Mehraban et al. [9] where only measurements of the RNFL in the peripapillary area were evaluated. In their study, Mehraban et al. [9] found significant changes in the superior, inferior, and nasal quadrants, failing to find any changes in the temporal area. Their nasal sector. The numbers show the level of significance; bold letters indicate $p<0.05$. a GCL + thickness. b GCL ++ thickness. c RNFL thickness. OCT optical coherence tomography, GCL ganglion cell layer, RNFL retinal nerve fiber layer. (color figure online)

results support our own findings, since none of the peripapillary retinal layers was affected in the temporal quadrant/ sector. However, and contrary to our results (as well as Mehraban's), Kalenderoglu and Khalil did find a significant reduction in the temporal sector $[7,8]$. We believe that the differences between these results might be due to the disparity in sample sizes and the OCT devices used (three different Spectral domain devices, and SS in our case). Our own results might have been limited by the small sample size. Additionally, we could not find any previously published study using SS technology in the analysis of the retina of BD patients; therefore, further studies using this new technology in a larger sample size are needed to corroborate our findings.

Axonal loss and decrease of the GCL has been found in other neurodegenerative diseases, such as multiple sclerosis $[13,14]$ and Parkinson's disease [12, 15]. Structural changes in these patients seem to appear not only as a result of retrograde neuronal loss, but also as a primary alteration $[16,17]$. Patients with psychiatric disorders have also been shown to have axonal loss quantifiable by OCT. Patients with schizophrenia showed a reduction of the RNFL and macular thickness, suggesting a neurodegenerative process underlying the physiopathological mechanism of the disease [4]. Fibromyalgia patients were demonstrated to have significant thinning of the RNFL thickness suggesting also the presence of possible neurodegeneration [3]. Our results, along with those of previous published studies on BD, seem to point out that neurodegeneration is also present in $\mathrm{BD}$ patients; this fact might be of importance in the differential diagnosis of $\mathrm{BD}$, since neurodegeneration as observed with OCT has not been demonstrated in patients with unipolar depression [18].

Contrary to patients with neurodegenerative diseases, changes in the choroidal plexus were not observed in our patients, despite a tendency toward choroidal thinning in 
BD patients. This might indicate that the pathophysiological mechanisms causing neurodegeneration in BD are different than those present in other neurological diseases, such as multiple sclerosis or Parkinson's [12, 19]. However, choroidal measurements obtained with Triton OCT show an elevated SD compared to retinal measurements. The small sample size included in the present study might be causing choroidal changes not to be detected in the statistical analysis.

A significant inverse correlation was found between disease duration and structural measurements (full macular thickness and peripapillary RNFL thickness) in our patients. A recent study [7] showed an inverse correlation between GCL volume and disease duration, disease scores and number of hospitalizations. However, Khalil et al. [8] did not find a significant correlation between similar disease features and OCT parameters. Again, variability in these results might be explained by different sample sizes and devices used in the studies. Effects like possible accelerated aging caused by BD or a possible predisposition to vascular changes in patients affected by BD have not been excluded as possible explanations for changes in retinal structures found in these patients. Pathophysiology of retinal changes in $\mathrm{BD}$ is yet to be evaluated and understood. Changes in choroidal thickness were not observed in our patients, however, a larger sample size might be needed to corroborate this finding. Also, further longitudinal studies should provide more information on retinal changes and its possible correlation to accelerated aging in these disease.

A well-known limitation for studies assessing psychiatric patients is the presence of smoking habit, since the prevalence of smoking in psychiatric disorders such as schizophrenia [20], BD [21], or major depression [22] is higher than the population's average. Progressive retinal changes caused by tobacco have been widely demonstrated. Smokers were shown to have a significant decrease of the peripapillary RNFL [23], foveal thickness [24], the ganglion cell complex [25], and a significant decrease in the blood flow index of the choriocapillary plexus in the macular area [26]. In order to avoid any bias caused by the smoking habit, we carefully analyzed our samples and ascertained that the number of smokers was not significantly different between the groups. Thus, we can conclude that the differences found in the retinal measurements between BD patients and controls in our study were not caused by the smoking habit.

In conclusion, our findings suggest that there is neuronal degeneration in the retina of BD patients. These patients present quantifiable thinning of the macular GCL, as well as a reduction of the peripapillary RNFL and GCL thickness. SS DRI Triton OCT detects structural changes in the different retinal layers in these patients, suggesting that the analysis of the retinal sublayers performed with this new technology might be a useful indicator to show degeneration and monitor disease progression in BD.

\section{Summary}

\section{What was known before}

- Bipolar disorder patients present retinal changes detectable with spectral domain OCT.

\section{What this study adds}

- This is the first study on the evaluation of retinal changes in patients with bipolar disorder using new swept source OCT technology.

- This study presents a complete analysis of full retinal thickness, RNFL, ganglion cell layer, and the choroidal plexus in these patients.

\section{Compliance with ethical standards}

Conflict of interest The authors declare that they have no conflict of interest.

\section{References}

1. Satue M, Rodrigo MJ, Otin S, Bambo MP, Fuertes MI, Ara JR, et al. Relationship between visual dysfunction and retinal changes in patients with multiple sclerosis. PLoS ONE. 2016;11: e0157293.

2. Polo V, Satue M, Rodrigo MJ, Otin S, Alarcia R, Bambo MP, et al. Visual dysfunction and its correlation with retinal changes in patients with Parkinson disease. BMJ Open. 2016;6:e009658.

3. Garcia-Martin E, Garcia-Campayo J, Puebla-Guedea M, Ascaso FJ, Roca M, Gutierrez-Ruiz F, et al. Fibromyalgia is correlated with retinal nerve fiber layer thinning. PLoS ONE. 2016;11: $\mathrm{e} 0161574$.

4. Yılmaz U, Küçük E, Ülgen A, Özköse A, Demircan S, Ulusoy $\mathrm{DM}$, et al. Retinal nerve fiber layer and macular thickness measurement in patients with schizophrenia. Eur J Ophthalmol. 2016;26:375-8.

5. Akiskal HS, Bourgeois ML, Angst J, Post R, Möller H, Hirschfeld $\mathrm{R}$. Re-evaluating the prevalence of and diagnostic composition within the broad clinical spectrum of bipolar disorders. J Affect Disord. 2000;59:S5-30.

6. Benazzi F. Bipolar II disorder: epidemiology, diagnosis and management. CNS Drugs. 2007;21:727-40.

7. Kalenderoglu A, Sevgi-Karadag A, Celik M, Egilmez OB, HanAlmis B, Ozen ME. Can the retinal ganglion cell layer (GCL) volume be a new marker to detect neurodegeneration in bipolar disorder? Compr Psychiatry. 2016;67:66-72.

8. Khalil MA, Saleh AA, Gohar SM, Khalil DH, Said M. Optical coherence tomography findings in patients with bipolar disorder. J Affect Disord. 2017;218:115-22.

9. Mehraban A, Samimi SM, Entezari M, Seifi MH, Nazari M, Yaseri M. Peripapillary retinal nerve fiber layer thickness in 
bipolar disorder. Graefes Arch Clin Exp Ophthalmol. 2016;254:365-71.

10. American Psychiatric Association. Diagnostic and statistical manual of mental disorders. 4th ed. Washington, DC: American Psychiatric Association; 2000.

11. González-López JJ, Rebolleda G, Leal M, Oblanca N, MuñozNegrete FJ, Costa-Frossard L, et al. Comparative diagnostic accuracy of ganglion cell-inner plexiform and retinal nerve fiber layer thickness measures by Cirrus and Spectralis optical coherence tomography in relapsing-remitting multiple sclerosis. Biomed Res Int. 2014;2014:128517.

12. Satue M, Obis J, Alarcia R, Orduna E, Rodrigo MJ, Vilades E, et al. Retinal and choroidal changes in patients with Parkinson's disease detected by swept source optical coherence tomography. Curr Eye Res. 2017;7:1-7.

13. Herrero R, Garcia-Martin E, Almarcegui C, Ara JR, RodriguezMena D, Martin J, et al. Progressive degeneration of the retinal nerve fiber layer in patients with multiple sclerosis. Invest Ophthalmol Vis Sci. 2012;53:8344-9.

14. Petzold A, Balcer LJ, Calabresi PA, Costello F, Frohman TC, Frohman EM, et al. Retinal layer segmentation in multiple sclerosis: a systematic review and meta-analysis. Lancet Neurol. 2017;16:797-812.

15. Satue M, Seral M, Otin S, Alarcia R, Herrero R, Bambo MP, et al. Retinal thinning and correlation with functional disability in Parkinson's disease patients. Br J Ophthalmol. 2014;98:350-5.

16. Lampert EJ, Andorra M, Torres-Torres R, Ortiz-Pérez S, Llufriu $\mathrm{S}$, Sepúlveda $\mathrm{M}$, et al. Color vision impairment in multiple sclerosis points to retinal ganglion cell damage. J Neurol. 2015;262:2491-7.

17. Bodis-Wollner I. Retinopathy in Parkinson disease. J Neural Transm. 2009;116:1493-501.

18. Sönmez İ, Köşger F, Aykan Ü. Retinal nerve fiber layer thickness measurement by spectral-domain optical coherence tomography in patients with major depressive disorder. Noro Psikiyatr Ars. 2017;54:62-6.

19. Esen E, Sizmaz S, Demir T, Demirkiran M, Unal I, Demircan N. Evaluation of choroidal vascular changes in patients with multiple sclerosis using enhanced depth imaging optical coherence tomography. Ophthalmologica. 2016;235:65-71.

20. De Leon J, Diaz FJ. A meta-analysis of worldwide studies demonstrates an association between schizophrenia and tobacco smoking behaviors. Schizophr Res. 2005;76:135-57.

21. Dickerson F, Stallings CR, Origoni AE, Vaughan C, Khushalani $\mathrm{S}$, Schroeder J, et al. Cigarette smoking among persons with schizophrenia or bipolar disorder in routine clinical settings. Psychiatr Serv. 2013;64:44-50.

22. Pratt LA, Brody DJ. Depression and smoking in the U.S. household population aged 20 and over, 2005-2008. NCHS Data Brief. 2010;34:1-8

23. El-Shazly AAE, Farweez YAT, Elewa LS, Elzankalony YA, Farweez BAT. Effect of active and passive smoking on retinal nerve fibre layer and ganglion cell complex. J Ophthalmol. 2017;2017:6354025.

24. El-Shazly AA, Farweez YA, Elzankalony YA, Elewa LS, Farweez BA. Effect of smoking on macular function and structure in active smokers versus passive smokers. Retina. 2018; 38:1031-1040

25. Moschos MM, Nitoda E, Laios K, Ladas DS, Chatziralli IP. The impact of chronic tobacco smoking on retinal and choroidal thickness in Greek population. Oxid Med Cell Longev. 2016;2016:2905789.

26. Ayhan Z, Kaya M, Ozturk T, Karti O, Hakan Oner F. Evaluation of macular perfusion in healthy smokers by using optical coherence tomography angiography. Ophthalmic Surg Lasers Imaging Retina. 2017;48:617-22. 